\title{
Results from Borexino on solar and geo-neutrinos
}

\author{
Daniele Guffanti*, on behalf of the Borexino Collaboration** \\ Gran Sasso Science Institute \& INFN Gran Sasso National Laboratories \\ E-mail: daniele.guffanti@gsi.it
}

** M. Agostini, K. Altenmüller, S. Appel, V. Atroshchenko, Z. Bagdasarian, D. Basilico,

G. Bellini, J. Benziger, G. Bonfini, D. Bravo, B. Caccianiga, F. Calaprice, A. Caminata,

L. Cappelli, S. Caprioli, M. Carlini, P. Cavalcante, F. Cavanna, A. Chepurnov, K. Choi,

L. Collica, D. D’Angelo, S. Davini, A. Derbin, X.F. Ding, A. Di Ludovico, L. Di Noto,

I. Drachnev, K. Fomenko, A. Formozov, D. Franco, F. Gabriele, C. Galbiati, M. Gschwender,

C. Ghiano, M. Giammarchi, A. Goretti, M. Gromov, D. Guffanti, T. Houdy, E. Hungerford,

Aldo Ianni, Andrea Ianni, A. Jany, D. Jeschke, S. Kumaran, V. Kobychev, G. Korga,

T. Lachenmaier, M. Laubenstein, E. Litvinovich, P. Lombardi, L. Ludhova, G. Lukyanchenko,

L. Lukyanchenko, I. Machulin, G. Manuzio, S. Marcocci, J. Maricic, J. Martyn, E. Meroni,

M. Meyer, L. Miramonti, M. Misiaszek, V. Muratova, B. Neumair, M. Nieslony, L. Oberauer,

V. Orekhov, F. Ortica, M. Pallavicini, L. Papp, Ö. Penek, L. Pietrofaccia, N. Pilipenko, A. Pocar,

A. Porcelli, G. Raikov, G. Ranucci, A. Razeto, A. Re, M. Redchuk, A. Romani, N. Rossi,

S. Rottenanger, S. Schönert, D. Semenov, M. Skorokhvatov, O. Smirnov, A. Sotnikov,

L.F.F. Stokes, Y. Suvorov, R. Tartaglia, G. Testera, J. Thurn, E. Unzhakov, A. Vishneva,

R.B. Vogelaar, F. von Feilitzsch, S. Weinz, M. Wojcik, M. Wurm, O. Zaimidoroga, S. Zavatarelli,

K. Zuber, G. Zuzel.

\begin{abstract}
Borexino is running at the INFN Gran Sasso National Laboraries, Italy since 2007. Its technical distinctive feature is the unprecedented ultralow background of the inner scintillating core, which is the foundation for the outstanding achievements accumulated by this experiment. In this contribution, after recalling the main features of the detector, the impressive solar data gathered so far by Borexino will be summarized. Special emphasis will be given to the illustration of the recent release of the fluxes as stemming from the simultaneous real time spectroscopy of the neutrinos from the entire $p p$ nuclear fusion chain in the Sun, opening with the remarkable $2.7 \%$ accuracy of the ${ }^{7} \mathrm{Be}$ flux the era of precision measurements also in the realm of the sub-MeV solar neutrinos. Such results put Borexino in the unique situation of being the only detector able to perform solar neutrino spectroscopy over the entire solar spectrum; the counterpart of this peculiar status, in the flavor conversion interpretation of the solar neutrino data, is the capability of Borexino alone to perform the full validation across the solar energy range of the MSW-LMA paradigm. This contribution will be concluded with an account of the Borexino accomplishments in the geo-neutrino field, marked by the detection of the geo-neutrino signal with a significance as high as $5.9 \sigma$.
\end{abstract}

The 39th International Conference on High Energy Physics (ICHEP2018)

4-11 July, 2018

Seoul, Korea

${ }^{*}$ Speaker. 


\section{Introduction}

Borexino is an ultrapure liquid scintillator experiment [1] installed at the underground INFN Gran Sasso National Laboratories, primarily devoted to the detection of solar neutrinos through their interactions with 278 ton of ultra pure liquid scintillator (LS). The LS, a mixture of pseudocumene (PC) with $1.5 \mathrm{~g} / 1$ of PPO, is contained into a $4.25 \mathrm{~m}$ radius, $125 \mu \mathrm{m}$ thick nylon Inner Vessel (IV) and constitutes the active volume (AV) of the detector, shielded from external $\gamma$-ray and neutron background by a quenched non-scintillating PC "buffer". The photons produced by particles interacting in the AV are detected with 2212 PMTs mounted on the surface of a 6.85 m radius Stainless Steel Sphere (SSS), yielding approximatively 500 p.e./MeV/(2000 PMTs). In addition to the energy deposited, the event position can also be reconstructed with a time-of-flight approach with $\sigma \approx 12 \mathrm{~cm}$ at $1 \mathrm{MeV}$. The SSS is contained in a cylindrical dome-shaped tank filled with water and equipped with 208 PMTs that provides an additional shielding to environmental radiation as well as an active veto system for residual cosmic muons. The low event rate of solar- $\nu$ s (a few tens counts per day (cpd) in 100 ton of LS) poses extreme requirements to the LS radioactive contaminations. The results of the experiments relies on the success of the LS purification, that reached the impressive level of $\lesssim 10^{-18} \mathrm{~g}\left({ }^{238} \mathrm{U},{ }^{232} \mathrm{Th}\right) / \mathrm{g}(\mathrm{LS})$ after the last purification campaign in 2010-2011.

\section{Solar neutrino precision measurements}

Most of solar- $v$ s are emitted at various stages of the proton-proton $(p p)$ fusion chain, the series of nuclear reactions that provides $\approx 99 \%$ of the Sun energy. The remaining $\approx 1 \%$ is due to the $\mathrm{CNO}$ cycle, a loop of reactions in which Carbon, Nitrogen and Oxygen catalyze the formation of a ${ }^{4} \mathrm{He}$ nucleus from 4 protons. While the overall solar- $v$ spectrum reach $16 \mathrm{MeV}$ the majority of the neutrino components have energy $<2 \mathrm{MeV}$ : because of the very different background affecting the measurement the Low Energy (LER, 0.19-2.93 MeV) and the High Energy Region (HER, 3.7$16 \mathrm{MeV}$ ) were treated separately.

The dataset used for the analysis of the LER[2, 3] was collected between December 2011 and May 2016. Muons crossing the inner (outer) detector are identified and a $300 \mathrm{~ms}$ ( $2 \mathrm{~ms}$ ) time window is vetoed to remove short-living cosmogenic background. Further background coming from radioactive contaminations of the IV, PMTs and of the SSS is reduced by applying a Fiducial Volume (FV) cut selecting the innermost part of the $\mathrm{AV}(r<2.8 \mathrm{~m},-1.8<z<2.2 \mathrm{~m}, 71.3 \mathrm{t}$ mass). Residual cosmogenic background due to the formation of ${ }^{11} \mathrm{C}\left(\beta^{+}\right)$can be reduced thanks to the so called Threefold Coincidence (TCF) veto, that exploits the space-time correlation of an event with a $\mu$ track and the detection of one or more neutrons that are often produced in the same process generating ${ }^{11} \mathrm{C}$.

A multivariate analysis is performed, fitting simultaneously the ${ }^{11} \mathrm{C}$-sub. and -tag. datasets to obtain all the low energy components of the solar- $v$ spectrum with a single fit in the LER. In addition to the energy spectrum the events radial distribution was considered to better identify external $\gamma$ 's background, as well as a pulse shaped parameter improving the discrimination of the residual ${ }^{11} \mathrm{C} \beta^{+}$events in the ${ }^{11} \mathrm{C}$-sub. spectrum [3].

The results are summarized in Tab. 1 [2]. The most abundant neutrinos produced by the Sun, the $p p$ neutrinos, are measured with $\approx 10 \%$ accuracy. The monochromatic ${ }^{7} \mathrm{Be}$ were measured 


\begin{tabular}{|c|c|c|c|c|}
\hline \multirow{2}{*}{ Solar $v$} & \multirow{2}{*}{$\begin{array}{c}\text { Rate } \\
(\mathrm{cpd} / 100 \mathrm{t})\end{array}$} & \multirow{2}{*}{$\begin{array}{c}\text { Flux } \\
\left(\mathrm{cm}^{-2} \mathrm{~s}^{-1}\right)\end{array}$} & \multicolumn{2}{|c|}{ Flux - SSM predictions $\left(\mathrm{cm}^{-2} \mathrm{~s}^{-1}\right)$} \\
\hline & & & $\mathrm{HZ}$ & $\mathrm{LZ}$ \\
\hline$p p$ & $134 \pm 10_{-10}^{+6}$ & $\left(6.1 \pm 0.5_{-0.5}^{+0.3}\right) \times 10^{10}$ & $5.98(1 \pm 0.006) \times 10^{10}$ & $6.03(1 \pm 0.005) \times 10^{10}$ \\
\hline${ }^{7} \mathrm{Be}$ & $48.3 \pm 1.1_{-0.7}^{+0.4}$ & $\left(4.99 \pm 0.11_{-0.08}^{+0.06}\right) \times 10^{9}$ & $4.93(1 \pm 0.06) \times 10^{9}$ & $4.50(1 \pm 0.06) \times 10^{9}$ \\
\hline рер (HZ) & $2.43 \pm 0.36_{-0.22}^{+0.15}$ & $\left(1.27 \pm 0.19_{-0.12}^{+0.08}\right) \times 10^{8}$ & \multirow{2}{*}{$1.44(1 \pm 0.01) \times 10^{8}$} & \multirow{2}{*}{$1.46(1 \pm 0.009) \times 10^{8}$} \\
\hline рер (LZ) & $2.65 \pm 0.36_{-0.24}^{+0.15}$ & $\left(1.39 \pm 0.19_{-0.13}^{+0.08}\right) \times 10^{8}$ & & \\
\hline${ }^{8} \mathrm{~B}$ & $0.223_{-0.016-0.006}^{+0.015+0.006}$ & $\left(5.68_{-0.41-0.03}^{+0.39+0.03}\right) \times 10^{6}$ & $5.46(1 \pm 0.12) \times 10^{6}$ & $4.50(1 \pm 0.12) \times 10^{6}$ \\
\hline $\mathrm{CNO}$ & $<8.1$ (95\% C.L.) & $<7.9 \times 10^{8}(95 \%$ C.L. $)$ & $4.88(1 \pm 0.11) \times 10^{8}$ & $3.51(1 \pm 0.10) \times 10^{8}$ \\
\hline hep & $<0.002$ (90\% C.L.) & $<2.2 \times 10^{5}$ (90\% C.L.) & $7.98(1 \pm 0.30) \times 10^{8}$ & $8.25(1 \pm 0.12) \times 10^{8}$ \\
\hline
\end{tabular}

Table 1: Measured interaction rate of solar- $v$ s. The calculated flux computed assuming the MSW-LMA oscillation parameter is reported next to the predictions of the SSMs.

with a remarkable $2.7 \%$ precision, twice as better as the theoretical prediction of the SSM. The pep neutrinos were also measured: their signal is correlated with the $v(\mathrm{CNO})$ one and with ${ }^{210} \mathrm{Bi}$. To break this correlation the CNO rate was constrained to the prediction of the Standard Solar Model (SSM) both for High and Low Metallicity (HZ, LZ) [4] and therefore two different values are reported. The analysis has no sensitivity to CNO neutrinos because of the above mentioned correlation with ${ }^{210} \mathrm{Bi}$ due to a very similar spectral shape: to improve the sensitivity the ratio between the $p p$ and $p e p$ neutrino fluxes was constrained since the two reactions share the same matrix element, obtaining an upper limit for the $\mathrm{CNO}$ flux $[2,3]$ that is consistent with the previous limit, also set by Borexino [5].

The low flux of $v\left({ }^{8} \mathrm{~B}\right)$ populating the HER demands a different analysis approach $[2,6]$. In order to maximise the exposure the analysis included data from the Phase I (January 2008) until December 2016. To avoid background due to radioactive contaminations, only events with $E>3.2 \mathrm{MeV}$ were selected, dividing the dataset in two regions: for events with energy larger than 5.7 MeV (HER-II) the entire AV was used, while for events with $3.2<E<5.7 \mathrm{MeV}$ (HER-I) a cut for $z<2.5 \mathrm{~m}$ was applied to contain the effect of a small leak of PPO in the non-scintillating buffer. Similarly to the low energy analysis, muons were removed and cosmogenic background was suppress by vetoing a time window after the passage of a muon. The ${ }^{8} \mathrm{~B}$ signal is extracted fitting the events radial distribution of the HER-I -II datasets independently: the main background were $\gamma$ rays from neutron capture in the SSS and in the PMTs and ${ }^{208} \mathrm{Tl}$ contaminations (in HER-I only). These measurements are combined in the value reported in Tab. 1 and, although not world-best, significantly improved the previous Borexino result [7].

This set of measurements makes Borexino the first experiment able to investigate solar- $v \mathrm{~s}$ throughout the entire $p p$ chain, while setting the best upper limit on the CNO neutrino flux. The results in Tab. 1 allow to test both neutrino properties and solar models. Assuming the fluxes predicted by the SSMs, the $v_{e}$ survival probability can be obtained in an energy range spanning from $100 \mathrm{keV}$ to $10 \mathrm{MeV}$, thus investigating both the vacuum-dominated and the matter-enhanced flavour conversion paradigm [2]. On the other hand, assuming the neutrino oscillation parameters it is possible to use these results to test the predictions of the SSM and tackle the current controversy on the solar abundances. A frequentist hypothesis test performed using the Borexino results on ${ }^{7} \mathrm{Be}$ and ${ }^{8} \mathrm{~B}$ fluxes showed a mild preference for the HZ SSM, but performing a global analysis 
including KamLAND, SNO, Super-Kamiokande and radiochemical experiments data considerably reduces the significance [2].

\section{Geo-neutrinos detection in Borexino}

The relatively large $\mathrm{AV}$, the outstanding radiopurity and the large distance from active nuclear power plants put Borexino in the ideal situation for detecting geo-neutrinos, i.e. those $\bar{v}_{e}$ produced by the decay of long lived isotopes present in the Earth. Geo-neutrinos are direct messengers of the abundances of radioactive elements and can provide extremely meaningful information about the radiogenic contribution to the total heath balance of the planet, discriminating different models of the Earth.

Geo-neutrinos can be detected via inverse $\beta$-decay, that present a distinctive signature given by the positron prompt energy deposit and subsequent annihilation followed by the characteristic 2.2 MeV $\gamma$ from neutron capture. The latest measurement released by Borexino [8] is based on data collected between December 2007 and March 2015. An un-binned likelihood fit was performed on the prompt energy spectrum of the 77 candidates to disentangle the geo- $v$ signal from the background, mostly due to reactor neutrinos. The considered exposure was not sufficient to resolve the ${ }^{238} \mathrm{U}$ and ${ }^{232} \mathrm{Th}$ separately, but when fixing their ratio according to the chondritic model, the evidence of the geo- $\nu$ detection exceeded for the first time the $5 \sigma$ threshold, reaching a $5.9 \sigma$ significance.

\section{Conclusion and outlooks}

The extreme level of radiopurity reached in the last purification campaigned allowed the Borexino to improve its previous results $[9,10]$. After the successful investigation of the $p p$-chain, the next main goal of the experiment is the search for $\mathrm{CNO}$ neutrinos: this task necessarily requires an independent determination of the ${ }^{210} \mathrm{Bi}$ background in the LS that is extremely challenging.

The results on geo-neutrinos can also be improved in the future benefiting from the increased exposure accumulated since the last data release.

\section{References}

[1] G. Alimonti et al. (Borexino Coll.), Nucl. Instr. Meth. Phys. Res. A, 600(3) (2009)

[2] M. Agostini et al. (Borexino Coll.), Nature, 562 (2018)

[3] M. Agostini et al. (Borexino Coll.), arXiv:1707.09279 [hep-ex] (2017)

[4] N. Vinyoles et al., Astrophys. J, 835(2) (2017)

[5] G. Bellini et al. (Borexino Coll.), Phys. Rev. Lett., 108 (5) (2012)

[6] M. Agostini et al. (Borexino Coll.), arXiv:1709.00756 [hep-ex] (2017)

[7] G. Bellini et al. (Borexino Coll.), Phys. Rev. D, 82 (3) (2010)

[8] M. Agostini et al., Phys. Rev. D, 92(3) (2015)

[9] G. Bellini et al. (Borexino Coll.), Phys. Rev. D, 89(11) (2014)

[10] G. Bellini et al. (Borexino Coll.), Nature, 512 (2014) 